\title{
Spectral properties of the Bose-Hubbard model within the cluster perturbation theory in $\mathrm{X}$-operators representation
}

\author{
Kirill Kudashkin $^{1}$ • Sergey Nikolaev ${ }^{1,2}$ - Sergey Ovchinnikov ${ }^{1,2}$
}

Received: date / Accepted: date

\begin{abstract}
We study the two-dimensional ultracold Bose gas in optical lattice. We use cluster perturbation theory based on Hubbard $X$-operators to calculate the spectral function and phase diagram of Bose-Hubbard model which is minimal model to describe behavior of ultracold gases in optical lattices. We have analyzed spectral properties of spinless bosons in a square lattice taking into account the short-range correlation.
\end{abstract}

Keywords Bose-Hubbard model · ultracold gases · cluster perturbation theory $\cdot$ density of states $\cdot$ spectral properties

PACS 05.30.Jp $\cdot$ 05.30.Rt $\cdot$ 64.70.Tg

\section{Introduction}

Ultracold gases have been studied intensively since the achievement of Bose-Einstein condensation. This achievement results in two major developments. One can tune the strengths of interaction between particles by means of Feshbach resonance and change the dimensionality with optical lattices. Using both of them or separately, one can enter a regime in which ultracold gas is no longer described by noninteracting quasiparticles. On contrary, such system will exhibit strong interactions and should be described adequately. Interest to ultracold gases in optical lattices comes from the possibility of studying phenomena of quantum many-body sys-

K. Kudashkin

E-mail: over9000nd@gmail.com

${ }^{1}$ Siberian Federal University, Krasnoyarsk, 660041, Russia

2 Kirensky Institute of Physics, Siberian Branch, Russian Academy of Sciences, Krasnoyarsk, 660036, Russia tems. Also ultracold systems could be used in quantum simulations [1,2]. Quantum phase transition from a superfluid to a Mott insulator has been realized in ultracold boson gases in optical lattices [3-6]. There have been done a lot of work in order to understand the behavior of this systems in different regimes [7-11].

In this paper we have studied ultracold spinless Bose particles in the optical lattice. A simple model to describe the behavior of such systems is Bose-Hubbard model [12]. We studied the Bose-Hubbard model with the nearest and next-to-nearest neighbor hoppings. We have used the cluster perturbation theory based on Hubbard $X$-operators [13] in order to acquire the spectral function. We calculate the phase diagram of the Mott insulator (MI) - superfluid state (SF) transition and determine the boundary of the two first Mott lobes for the different value of the next-to-nearest neighbor hoppings. We also study the density of states and the momentum distribution in the Mott phase taking into account the quasiparticle spectral weight.

The paper is organized as follows. Firstly, we introduce the Bose-Hubbard model with the next-to-nearest neighbor hoppings and describe the cluster perturbation theory based on $X$-operators in Section 2. Spectral properties and phase diagram of ultracold spinless bosons in the square optical lattice are described in Section 3. Finally, in Section 4 we draw the conclusion.

\section{Cluster perturbation theory based on $X$-operators}

A conceptually simple model to describe ultracold atoms in an optical lattice at finite density is obtained by combining the kinetic energy in the lowest band with the on-site repulsion in the limit of sufficiently deep optical 
lattice [7]. We study the Bose-Hubbard model with the next-to-nearest neighbor hoppings $J^{\prime}$ :

$$
\begin{gathered}
\hat{H}=-\sum_{<i, j>} J_{i j} \hat{b}_{i}^{\dagger} \hat{b}_{j}-\sum_{\ll i, j \gg} J_{i j}^{\prime} \hat{b}_{i}^{\dagger} \hat{b}_{j}+ \\
\frac{U}{2} \sum_{i} \hat{n}_{i}\left(\hat{n}_{i}-1\right)-\mu \sum_{i} \hat{n}_{i},
\end{gathered}
$$

where $\hat{b}_{i}^{\dagger}$ and $\hat{b}_{j}$ are creation and annihilation operators, respectively; $\hat{n}_{i}=\hat{b}_{i}^{\dagger} \hat{b}_{i}$ is the particle number operator, $U$ is the on-site repulsion energy; $J_{i j}$ is the hopping matrix element of nearest neighbors, $J_{i j}^{\prime}$ is the hopping matrix element of next-to-nearest neighbors; $\mu$ is the chemical potential. Here $\langle\ldots\rangle$ and $\ll \ldots \gg$ denote sum over nearest neighbors and sum over nextto-nearest neighbors, respectively. It is schematically shown in Figure 1.

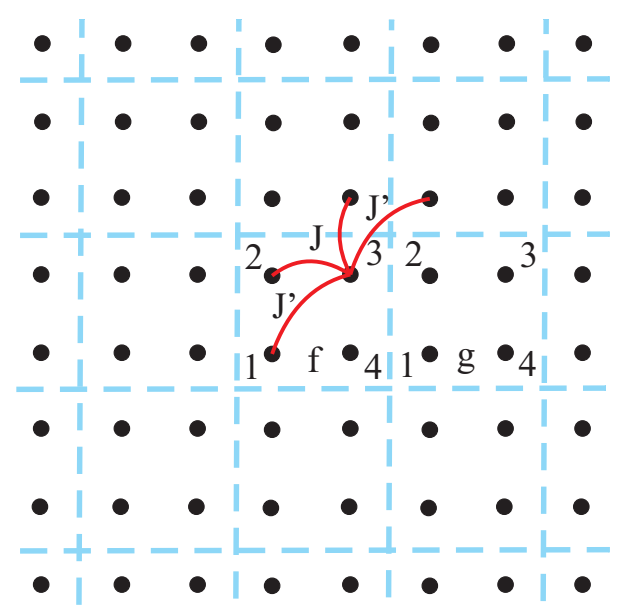

Fig. 1 Scheme of a square optical lattice. $J$ denotes the nearest neighbor hoppings, $J^{\prime}$ denotes the next-to-nearest neighbor hoppings. $f$ and $g$ are the cluster indexes.

In order to obtain spectral properties of ultracold gas in the optical lattice we used the cluster perturbation theory based on Hubbard $X$-operators [13]. Here we use the $2 \times 2$ cluster for tiling of the square lattice (see Figure 1).

Firstly, we divide Hamiltonian (1) into two part:

$\hat{H}=\sum_{f} \hat{H}_{0}(f)+\sum_{f \neq g} \hat{H}_{i n t}(f, g)$,

where $\hat{H}_{0}(f)$ is Hamiltonian of $2 \times 2$ cluster $f, \hat{H}_{i n t}(f, g)$ is the interaction between clusters $f$ and $g$.

Secondly, we exactly diagonalize $\hat{H}_{0}(f)$. Eigenvectors are used to construct X-operators [13-15]:

$\hat{X}_{f}^{\alpha} \equiv \hat{X}_{f}^{p q}=|p\rangle\langle q|$ where $|p\rangle$ is the initial cluster state, $\langle q|$ is the final cluster state, $\alpha(p, q)$ is a root vector of transition between states in the Hilbert space of cluster.

Now we can represent annihilation operator $\hat{b}_{i}$ in the form:

$\hat{b}_{i}=\sum_{f \alpha} \gamma_{i}(\alpha) \hat{X}_{f}^{\alpha}$

$\gamma_{i}(\alpha)=\left\langle m^{\prime}, N-1\left|\hat{b}_{i}\right| m, N\right\rangle$

where $i$ is index inside of a cluster and runs over 1 , $2,3,4, \gamma_{i}(\alpha)$ is the matrix element which determine a transition from state $|m, N\rangle$ to $\left|m^{\prime}, N-1\right\rangle, m$ and $m^{\prime}$ denote states in the Hilbert space with $N$ and $N-1$ particles, respectively.

Using commutation relation between Bose-operators, one can get the following sum rule:

$\left\langle\left[\hat{b}_{i}, \hat{b}_{i}^{\dagger}\right]\right\rangle=1=\sum_{\alpha}\left|\gamma_{i}(\alpha)\right|^{2} B(\alpha) \equiv f$,

$B(\alpha)=\left\langle X^{p p}\right\rangle-\left\langle X^{q q}\right\rangle$,

where $B(\alpha)$ is a filling factor [13]. In exact calculations including all possible quasiparticle excitations $\alpha$ the sum in Eq.(6) is equal to unity. Practically some part of the Hilbert space gives miserable contribution to the spectral function and may be omitted to reduce the computation time. Here we introduced $f$-factor which controls the sum over all transitions in the Hilbert space. This study shows that if one keeps this number within a range from 0.99 to 1 results will remain correct. This fact is only natural, we neglected unlikely transitions which didn't change a whole picture. More details on this matter one can find below.

Substituting (4) into (1) and keeping in mind that $\hat{H}_{0}(f)$ is diagonalized, we have:

$\hat{H}=\sum_{f n} \epsilon_{n} X_{f}^{n n}+\sum_{f \neq g} \sum_{\alpha \beta} J_{f g}^{\alpha \beta} X_{f}^{\alpha} X_{g}^{-\beta}$,

where $f$ and $g$ are cluster indexes, $\alpha$ and $\beta$ are root vectors of transitions between cluster states, $\epsilon_{n}$ is the energy of cluster, $J_{f g}^{\alpha \beta}$ is the matrix element of the interaction between clusters $f$ and $g$.

Let us carry out a Fourier transformation of $X$ operators. We take into account that these operators are defined for the cluster forming quadratic superlattice with a period twice as large as that of the initial lattice. In our case, the number of sites in a cluster is four. Hence, the total number of clusters is smaller than the initial number of sites also by a factor of four. Keeping this in mind, we have:

$X_{f}^{\alpha}=\frac{1}{\sqrt{N^{*}}} \sum_{\tilde{\mathbf{k}}} X_{\tilde{\mathbf{k}}}^{\alpha} e^{i \tilde{\mathbf{k}} \mathbf{R}_{f}}$, 
where $N^{*}$ is the number of clusters in the superlattice and $\tilde{\mathbf{k}}$ is a wave vector which runs over the reduced Brillouin zone. Taking into account (9), we obtain:

$\hat{H}_{J}=\sum_{\tilde{\mathbf{k}}} \sum_{\alpha, \beta} J_{\alpha \beta}(\tilde{\mathbf{k}}) \hat{X}_{\tilde{\mathbf{k}}}^{\alpha} \hat{X}_{\tilde{\mathbf{k}}}^{-\beta}$,

where $J_{\alpha \beta}(\tilde{\mathbf{k}})$ is the interaction between clusters.

To calculate the spectral function we used the twotime Green functions for the initial Bose-operators and for Hubbard bosons in the representation of $X$-operators:

$G\left(i f, t ; j g, t^{\prime}\right)=\left\langle\left\langle\hat{b}_{i f}(t) \mid \hat{b}_{j g}^{\dagger}\left(t^{\prime}\right)\right\rangle\right\rangle$,

$D_{\alpha \beta}\left(f, t ; g, t^{\prime}\right)=\left\langle\left\langle X_{f}^{\alpha}(t) \mid X_{g}^{\beta}\left(t^{\prime}\right)\right\rangle\right\rangle$,

where $i$ and $j$ are intra-cluster indexes, $f$ and $g$ are cluster indexes. Here we used notation from [13].

After writing the equations of motion for the Green function (12) and using the Fourier transform (9), we can obtain the following matrix equation in the HubbardI approximation:

$D^{-1}(\tilde{\mathbf{k}}, \omega)=\left(D_{0}(\omega)\right)^{-1}-J(\tilde{\mathbf{k}})$,

where

$D_{0}(\omega)=\frac{B(\alpha)}{\omega-\Omega(\alpha)} \delta_{\alpha \beta}$,

$\Omega(\alpha)=\epsilon_{q}(N+1)-\epsilon_{p}(N)$.

Expression (14) defines the cluster Green function which can be evaluated precisely and contains the energy (quasiparticle energy) of transition from the state $q$ with number of particles $N+1$ to the state $p$ with number of particles $N ; B(\alpha)$ is the filling factor (7).

Let us perform a Fourier transformation for Boseoperators:

$\hat{b}_{i f}=\frac{1}{\sqrt{N_{0}}} \sum_{\mathbf{k}} \bar{b}_{\mathbf{k}} \exp \left(i \mathbf{k} \cdot\left(\mathbf{R}_{f}+\mathbf{r}_{i}\right)\right)$,

where $N_{0}$ is the number of sites in the initial lattice and $\mathbf{k}$ is a wave vector defined in the initial Brillouin zone. There is a relation between Fourier transform of the Green functions (11) and (12):

$$
\begin{aligned}
G(\mathbf{k}, \omega)=\frac{1}{N_{c}} & \sum_{\alpha \beta} \sum_{i, j=1}^{N_{c}} \gamma_{i}(\alpha) \gamma_{j}^{*}(\beta) \times \\
& \times D_{\alpha \beta}(\mathbf{k}, \omega) e^{-i \mathbf{k}\left(\mathbf{r}_{i}-\mathbf{r}_{j}\right)},
\end{aligned}
$$

where $N_{c}$ is a number of sites in cluster (which is four in our case).

We using the following spectral function:

$A(\mathbf{k}, \omega)=-2 \operatorname{Im}[G(\mathbf{k}, \omega)]$
It is well known that the spectral function must obey the sum rule obtained from the commutation relation for Bose-operators as follows:

$$
\int d \omega A(\mathbf{k}, \omega)=\left\langle\left[\hat{b}_{\mathbf{k}}, \hat{b}_{\mathbf{k}}^{\dagger}\right]\right\rangle=1
$$

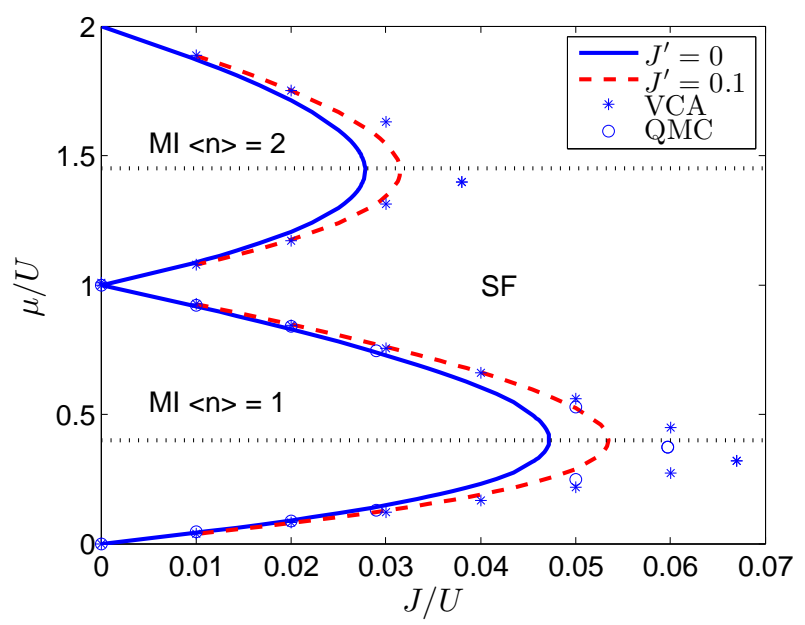

Fig. 2 The phase diagram of the Mott insulator (MI) - superfluid state (SF) transition. The first two Mott lobes with an occupation number per site $\langle n\rangle=1$ and $\langle n\rangle=2$ are presented. Solid blue line is acquired when the next-tonearest neighbor hoppings are disable $\left(J^{\prime}=0\right)$. Dashed red line is acquired when the next-to-nearest neighbor hoppings are enabled $\left(J^{\prime}=0.1 J\right)$. Asterisks denote the results of the variational cluster approach (VCA) [9] and circles denote the results of the quantum Monte-Carlo (QMC) [11].

Above, we introduced the concept of $f$-factor (6) which defines the extent to which equality equation (19) is satisfied, i.e.,

$\int d \omega A(\mathbf{k}, \omega)=f$

We can now fully estimate the necessity of monitoring $f$-factor at first steps of computation of the complete Green function. After exact diagonalization of the cluster and before calculating of the Green function we can choose this number of Bose quasiparticles which allow us to obey the sum rule at the end of calculations. In ideal case, we must include all transitions in Hilbert space. However, since we perform numerical calculations in which an increase in number of transition involves an increase the computation time. It is very important to determine the optimal number of transitions before calculations without noticeably affecting the final result. This is ensured by controlling $f$-factor at the beginning of numerical calculations. 

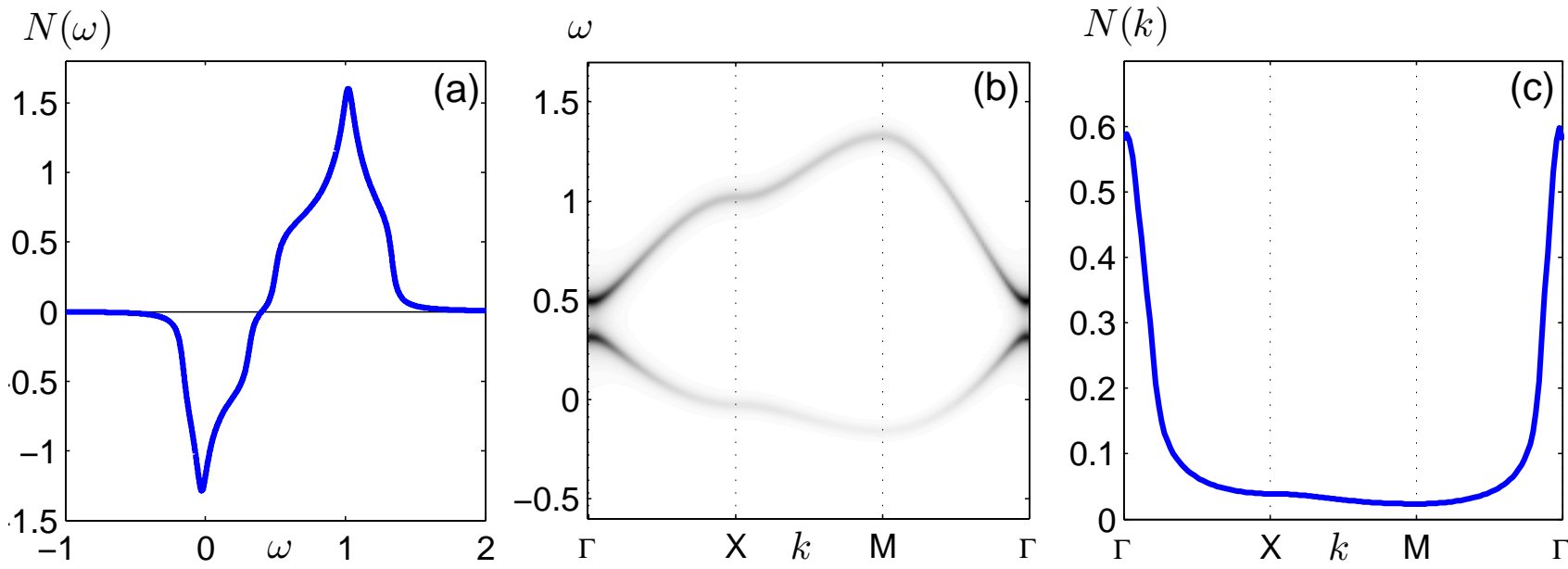

Fig. 3 (a) The density of states $N(\omega)$, (b) the dispersion $\omega(\mathbf{k})$, and (c) the momentum distribution $N(\mathbf{k})$. Here and below we use the following notation: $\Gamma=(0,0), X=(\pi, 0), M=(\pi, \pi)$. The hopping parameters $J / U=1 / 22$ and $J^{\prime}=0$.
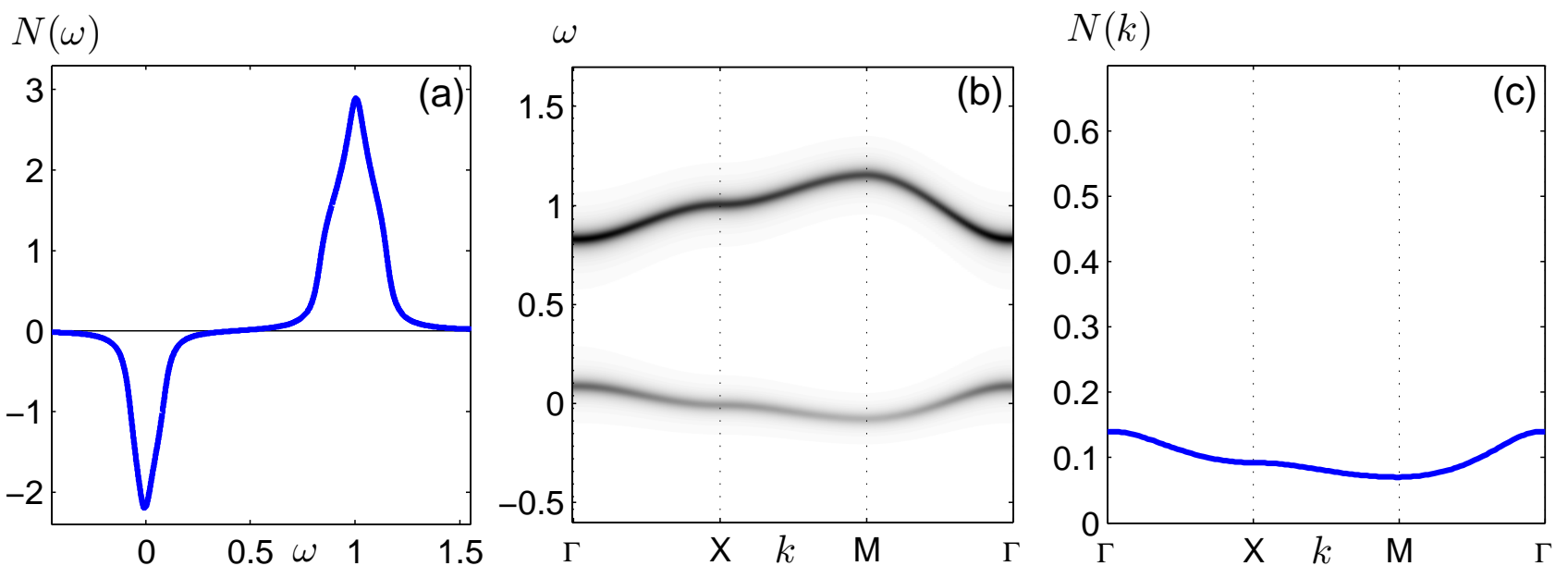

Fig. 4 The same as in Figure 3 for the hopping parameters $J / U=1 / 50$ and $J^{\prime}=0$.

\section{Spectral properties of the spinless bosons in the square lattice}

Here we study ultracold spinless Bose gas in the square optical lattice. For analyzing of the physical properties of this system in Mott phase we calculate the quasiparticle dispersion with spectral weight. In accordance with definition (13) the dispersion equation takes the form:

$\operatorname{det}\left(\left(\omega-\Omega(\alpha) \delta_{\alpha \beta}\right)-B(\alpha) J_{\alpha \beta}(\tilde{\mathbf{k}})\right)=0$.

The solution of this equation gives a bare dispersion for all quasiparticles. This set contains both dispersions that define bands as well as dispersionless levels, i.e., equation (21) gives such poles which can be cancelled out by $f$-factor. The spectral function (18) determines the spectral weight of all such states for each value of a wave vector and, thus, selects physically significant poles.

The Bose-Hubbard model exhibits a quantum phase transition from a Mott phase to a superfluid phase by changing an occupation number per site or increasing the ration $J / U$. The first two lobes of $2 \mathrm{D}$ Bose-Hubbard model obtained by means of the cluster perturbation theory based on X-operators are shown in Figure 2. We used a gap between bands to draw boundaries of the Mott phase on this phase diagram.

The critical ration is $(J / U)_{c}=0.047$ for the first Mott lobe and $(J / U)_{c}=0.028$ for the second Mott lobe when the next-to-nearest neighbor hoppings are disabled. For a case when the next-to-nearest neighbor hoppings are enabled $(J / U)_{c}=0.053$ for the first lobe and $(J / U)_{c}=0.032$ for the second one. Thus the Mott lobes increase with the next-to-nearest neighbor hopping $J^{\prime}$. The strong-coupling perturbation theory [16] 
and QMC [11] results $(J / U)_{c}=0.0597$ for the first lobe and $(J / U)_{c}=0.0378$ for the second one.

The dispersion $\omega(\mathbf{k})$, the density of states $N(\omega)$ and the momentum distribution $N(\mathbf{k})$ for an occupation number per site $\langle n\rangle=1$ and the nearest neighbor hopping $J / U=1 / 22$ are shown in Figure 3. In the numerical computation of spectral density we replaced a delta-function by a Lorenz curve with a broadening parameter $\delta=0.03$. All calculation was carried out while keeping $f$-factor at 0.995 . The dispersion is displayed along the symmetrical directions $\Gamma \rightarrow X \rightarrow M \rightarrow \Gamma$ in the first quadrant of the first Brillouin zone. We use the following notation $\Gamma=(0,0), X=(\pi, 0), M=(\pi, \pi)$.

An interesting feature of Bose systems is negative value of $N(\omega)$. It is result of the Green function definition [9]. When the strength of hoppings $J$ is growing in comparison with on-site interaction $U$ the gap between bands tend to narrow and close at critical $(J / U)_{c}$ (see Figures 3 and 4).

Particles tend to occupy sites instead of hopping when the nearest neighbor hopping $J$ are small. In this case the distribution of the spectral weight becomes more uniform and the momentum distribution $N(\mathbf{k})$ straightens (Figure 4b, c). For large hopping we see a peak in the momentum distribution at the $\Gamma$ point which is a precursor for the Bose-Einstein condensation. Figure $3 \mathrm{~b}$ shows the nonuniform distribution of the spectral weight in the dispersion. We can see a large spectral weight near the $\Gamma$ point. These results agree with the results obtained by means of the variational cluster approach [9] and the strong-coupling perturbation theory [16].

In addition, we studied and analyzed the spectral properties for $J^{\prime}=0.1 J$. But we don't present these results here because they doesn't qualitatively differ from results above.

\section{Conclusions}

In this paper, we have presented and discussed results obtained within the cluster perturbation theory based on Hubbard $X$-operators for the spectral properties of the two-dimensional Bose gas in optical lattice. The minimal model to describe the behavior of such system is the Bose-Hubbard model. It undergoes a quantum phase transition form the Mott phase to superfluid phase, depending on a occupation number per site and the ration $J / U$. We determined the first two Mott lobes of the phase diagram and obtained the change of boundary of the Mott phase with the next-to-nearest neighbor hopping $J^{\prime}$. In particular, the Mott lobes increase with $J^{\prime}$ (see Figure 2). Our results is in reasonable agreement with different methods for the boundaries of the Mott lobes apart from the region close to the tip. Despite the fact that methods like the quantum Monte-Carlo or the strong-coupling perturbation theory are very accurate, our approach requires less computational effort.

Moreover, we calculated the spectral function in the first Mott lobe of the phase diagram. An important fact about the cluster perturbation theory is that one can calculate the Green function in the real frequency domain and, thus, directly calculate the spectral function. It's shown that the distribution of the spectral weight is the uniform for a small nearest neighbor hopping $J$ and the sharply nonuniform for a large $J$. In particular, the spectral weight in the $\Gamma$ point increases with $J$ and, thus, it indicates a precursor to the Bose-Einstein condensation. We also calculated the density of states and the momentum distribution taking into account the quasiparticle spectral weight.

Acknowledgements This work was supported by RFBR grant 16-02-00098, Government of Krasnoyarsk Territory and RFBR according to the research projects 16-42-243048, 16-42-240511, and 16-42-240769, and the Russian President Grant NSh7559.2016 .2 .

\section{References}

1. T.H. Johnson, S.R. Clark, D. Jaksch, EPJ Quantum Technology 1, 1 (2014)

2. I. Buluta, F. Nori, Science 326, 108 (2009)

3. D. Jaksch, C. Bruder, J. Cirac, C. Gardiner, P. Zoller, Phys. Rev. Lett. 81, 3108 (1998)

4. M. Greiner, O. Mandel, T. Esslinger, T.W. Hänsch, I. Bloch, Nature 415, 39 (2002)

5. I.B. Spielman, W.D. Phillips, J.V. Porto, Physical Review Letters 98, 080404 (2007)

6. I.B. Spielman, W.D. Phillips, J.V. Porto, Physical Review Letters 100, 120402 (2008)

7. I. Bloch, D. Jean, Z. Wilhelm, Review of Modern Physics 80, 885 (2008)

8. L. Jiang, A.M. Rey, O. Romero-isart, J.J. García-ripoll, A. Sanpera, M.D. Lukin, Physical Review A 79, 022309 (2009)

9. M. Knap, E. Arrigoni, W.V.D. Linden, Physical Review B 81, 024301 (2010)

10. X.h. Zhang, S.p. Kou, International Journal of Modern Physics B 27(4), 1250214 (2013)

11. B. Capogrosso-Sansone, S.G. Soyler, N. Prokof'ev, B. Svistunov, Phys. Rev. A 77, 015602 (2008)

12. M.P.A. Fisher, P.B. Weichman, G. Grinstein, D.S. Fisher, Phys. Rev. B 40, 546 (1989)

13. S.V. Nikolaev, S.G. Ovchinnikov, Journal of Experimental and Theoretical Physics 111(4), 635 (2010)

14. J. Hubbard, Proceedings of the Royal Society A: Mathematical, Physical and Engineering Sciences 276, 238 (1963)

15. S. Ovchinnikov, V. Val'kov, Hubbard operators in the theory of strongly correlated electrons (Imperial College Press, London, 2004)

16. N. Elstner, H. Monien, Physical Review B 59(9), 12184 (1999) 